\title{
Modelling vertical concentration distributions of solids suspended in turbulent visco-plastic fluid
}

\author{
Václav Matoušek ${ }^{1 *}$, Andrew Chryss ${ }^{2}$, Lionel Pullum ${ }^{3}$ \\ ${ }^{1}$ Czech Technical University in Prague, Faculty of Civil Engineering, Department of Hydraulics and Hydrology, Thákurova 7, 16629 \\ Praha 6, Czech Republic. E-mail: v.matousek@fsv.cvut.cz \\ ${ }^{2}$ CSIRO Mineral Resources, Bayview Avenue, Clayton, Victoria 3168, Australia. E-mail: andrew.chryss@csiro.au \\ 329 Monash Avenue, Olinda, Victoria 3788, Australia. E-mail: lpullum@bigpond.net.au \\ * Corresponding author. E-mail: v.matousek@fsv.cvut.cz
}

\begin{abstract}
Vertical concentration distributions of solids conveyed in Newtonian fluids can be modelled using RouseSchmidt type distributions. Observations of solids conveyed in turbulent low Reynolds number visco-plastic carriers, suggest that solids are more readily suspended than their Newtonian counterparts, producing higher concentrations in the centre of the pipe. A Newtonian concentration profile model was adapted to include typical turbulent viscosity distributions within the pipe and particle motion calculated using non-Newtonian sheared settling. Predictions from this and the unmodified model, using the same wall viscosity, are compared with the chord averaged profile extracted from tomographic data obtained using a $50 \mathrm{~mm}$ horizontal pipe.
\end{abstract}

Keywords: Complex slurry flow; Non-Newtonian flow; Solids distribution; Pipe flow; Particle settling; Laboratory experiment.

\section{INTRODUCTION}

Industrial hydraulic conveying systems generally fall into two modes: highly turbulent Newtonian systems, where solids are suspended to some degree through interaction between the particles and turbulent eddies, and laminar non-Newtonian flows which behave either as homogeneous fluids or, where coarse particles are included, as sliding beds of these coarser solids immersed in a non-Newtonian carrier. In the latter case the highly viscous nature of the carrier fluids generally prevents turbulent instabilities from developing. For less viscous nonNewtonian carrier fluids low Reynolds number turbulence can be achieved and where coarser solids are included, these are suspended and transported in a similar manner to the ubiquitous turbulent Newtonian systems. Examples of such systems are low viscosity thickened discharge, typical of many modern waste disposal systems, and ash disposal systems where bottom ashes are added to a fly ash carrier slurry.

The similarity between the Newtonian and non-Newtonian turbulent systems suggests that existing methods based on Newtonian carrier fluids, almost exclusively water, should apply to these moderately viscous non-Newtonian systems. Turbulent pipeline system curves for Newtonian and nonNewtonian fluids are very similar and are successfully modelled with only relatively minor modifications to standard Newtonian formulations, (e.g., Wilson and Thomas, 1985). However, these are the gross characteristics of the pipeline flow and experiments using direct numerical simulation (DNS) and large eddy simulation (LES) (Gnambode et al., 2015; Rudman and Blackburn, 2006; Rudman et al., 2004; Singh et al., 2016) have shown that the internal structure of the non-Newtonian flows differs from their Newtonian counterparts in several important ways. In particular, the time averaged viscosity of the fluid increases towards the centre of the pipe, and when the fluid is visco-plastic and exhibits a yield stress, this effect becomes very pronounced confining the high energy eddies to a narrower annular region than for the Newtonian systems. It is expected that such behaviour would impact on the ability of the carrier fluids to suspend any solids too large, known simply as particles from here on, to form part of the carrier slurry, and how once suspended these particles might be distributed inside the pipe.

Observations in the laboratory using visco-plastic fluids conveying sand and gravel like particles in relatively small pipes $(50 \mathrm{~mm})$ indicate that the particles very rapidly move from sliding beds into pseudo-homogeneous suspension once the flow becomes turbulent, similar to the anomalous regime noted by Turian and Yuan (1977). Such behaviour was not entirely unexpected given that the viscosity of the fluid surrounding the particles is quite high and their settling velocities are lower than in water. However, it should also be stressed that these turbulent flows are low Reynolds number flows, where the eddy strengths are not as high.

One way to assess the similarity between these mildly nonNewtonian turbulent suspensions and the suspending capabilities of equivalent Newtonian suspensions might be to examine the extent and nature of the suspension through modelling of the vertical concentration distributions.

\section{CONCENTRATION DISTRIBUTION CALCULATION}

The chord averaged vertical distribution of particles in a pipe undergoing turbulent flow can be approximated using a onedimensional Rouse-Schmidt model (e.g., Eskin, 2005; Eskin 2012; Gillies, 1993; Gillies and Shook, 1994; Kaushal et al., 2002). For illustration purposes the Eskin model (Eskin, 2012) will be considered here.

\section{Basic method}

The full details of these models can be found by consulting the literature cited, but a generalized computational procedure is as follows. 
The one-dimensional advection-diffusion equation for particles suspended in a horizontal flow (e.g., Shook and Roco, 1991) is given by

$\frac{d c}{d y}=\frac{v_{t} c(1-c)^{m}}{\varepsilon_{s}(c)}$

where generally $v_{t}$ is the terminal settling velocity of a single particle at coordinate $y$, the distance from the bottom of the pipe, $c$ is the local particle volumetric concentration at $y, m$ a hindered settling exponent, and $\varepsilon_{s}(c)$ the particle diffusivity at $y$. This equation is iteratively integrated across the vertical diameter of the pipe until the average calculated concentration, given by

$$
C_{v}=\frac{2}{A} \int_{0}^{D} c \sqrt{y(D-y)} d y
$$

where, $A$ is the cross-sectional area of the pipe and $D$ is the pipe diameter, agrees with the actual average concentration to within the desired tolerance.

Before being able to perform these calculations for nonNewtonian suspension flows, however, three questions need to be answered

1. How do particles settle in sheared non-Newtonian fluids?

2. If and how do the carrier fluid properties vary spatially?

3. What is the particle diffusivity of particles suspended in turbulent non-Newtonian fluids?

\section{Sheared settling behaviour}

Unlike particle settling in Newtonian fluids, the settling behaviour of particles in non-Newtonian fluids differs depending upon the extent of the local shear field, or strain rate. A graphic demonstration of this occurs when particles suspended in a visco-plastic fluid with a sufficiently high yield stress may not settle while the fluid is undisturbed but fall as soon as the fluid surrounding the particle is sheared, by stirring for example. To understand this better, experiments were conducted at CSIRO in Melbourne monitoring the steady state fall velocity of single particles in sheared non-Newtonian fluids.

\section{Particle settling velocity}

A large volume vessel with counter rotating belts (see Figure 1) created a region of planar Couette flow. Spherical particles of differing diameters and densities were dropped down the central plane between the belts where zero axial translational velocity occurs. The axial direction is parallel to the belts and shown as " $u$ " in Figure 1. High speed video in the axial direction was used to monitor particle position from which fall velocity was calculated.

The belts are fully submerged in a well-defined nonNewtonian liquid and set in motion, with each belt having an equal and opposite velocity. One high and one low viscosity yield stress fluid (Carbopol 980) and a viscous fluid without yield stress $(\mathrm{CMC})$ were used as carrier fluids and their measured rheology is shown in Figure 2 and Table 1. The particle characteristics are shown in Table 2. For settling in quiescent fluids the close proximity of the walls affects the settling behaviour, and several authors, (e.g., Missirlis et al., 2001), have suggested that, for cylindrical settling chambers, the particle diameter to gap ratio should be no greater than $5 \%$ to ensure that the falling velocity is within $90 \%$ of the unhindered terminal settling velocity. Assuming similar conditions for sheared flow the maximum particle size that will meet this criterion varies from 1 to $9 \mathrm{~mm}$ depending on the gap between the belts.
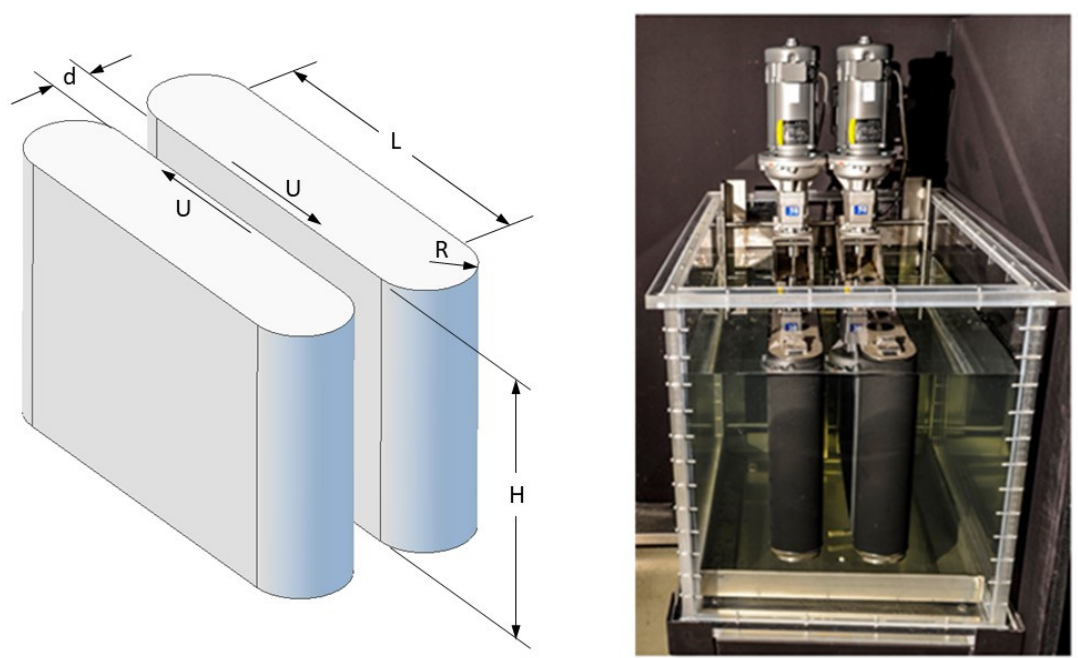

Fig. 1. Schematic of shear settling rig geometry (left) and photograph of sheared settling rig (right).

Table 1. Fluids used in sheared settling experiments.

\begin{tabular}{|c|c|c|c|c|c|c|}
\hline Material & $C_{w}(\%)$ & Fluid model & $\tau_{y}(\mathrm{~Pa})$ & & $k\left(\mathrm{~Pa} \mathrm{~s}^{\mathrm{n}}\right)$ & $n(-)$ \\
\hline Carbopol 980 & 0.1 & Herschel-Bulkley & 10 & & 2.19 & 0.50 \\
\hline Carbopol 980 & 0.15 & Herschel-Bulkley & 33 & & 13.1 & 0.37 \\
\hline Material & $C_{w}(\%)$ & Fluid model & $\eta_{w}(\mathrm{~Pa} \mathrm{~s})$ & $\eta_{\infty}(\mathrm{Pa} \mathrm{s})$ & $k\left(\mathrm{~Pa} \mathrm{~s}^{\mathrm{n}}\right)$ & $n(-)$ \\
\hline CMC & 0.6 & Cross model (Cross, 1965) & 0.64 & 0.001 & 0.3 & 0.53 \\
\hline
\end{tabular}

$\eta_{\infty}$ is the asymptotic viscosity as the particle approaches its terminal velocity 
Table 2. Spherical particles used in settling experiments.

\begin{tabular}{l|ll}
\hline Material & $\rho\left(\mathrm{kg} \mathrm{m}^{-3}\right)$ & $d_{p}(\mathrm{~mm})$ \\
\hline Glass & 2473 & 5.9 \\
Glass & 2750 & 8.5 \\
Ceramic & 2773 & 19 \\
Steel & 8042 & 3.96 \\
Steel & 8042 & 4.7 \\
Zirconia (PSZ) & 5904 & 7.6 \\
PVC & 1653 & $1.5-2.5$ \\
\hline
\end{tabular}

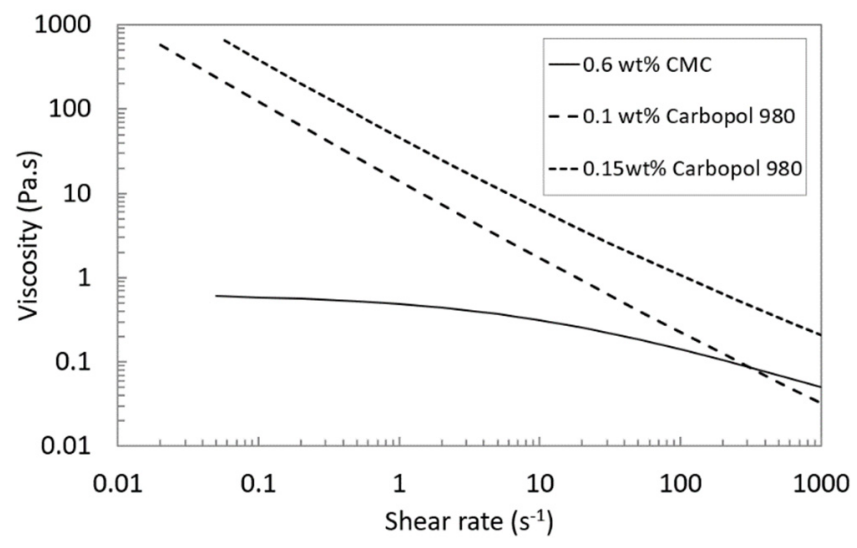

Fig. 2. Rheology of suspending media.

The accepted transitional energy gradient, $K$, for plane Couette flow of Newtonian fluids is of the order $K=370$ (Dou et al., 2007), and $K$ in this particular case reduces to a simple Reynolds number. Particle Image Velocimetry (PIV) in the horizontal mid-plane orientation of the shear rig has shown the stability of the flow streamlines and the extent of the useful measuring zone. The velocity measurements for $0.6 \mathrm{wt} \% \mathrm{CMC}$ are shown in Figure 3, where the wall Reynolds number is $R e=\frac{\rho_{f} u d}{\eta_{W}}$ in which $\rho_{f}$ is the fluid density, $u$ is the wall velocity, $d$ is the gap width and $\eta_{w}$ is the viscosity at the wall. The Re variation is achieved by altering the gap between the belts from 130 to $200 \mathrm{~mm}$. It can be seen that a stable region exists in the centre of the belts for $R e=230$, but significant variations occur for $R e>300$ in keeping with preliminary CFD analysis. Fall velocity measurements were conducted with this in mind. Some minor entrance and exit effects were also noted and these regions avoided for testing.

The experimental results are shown in Figure 4 and compared to Newtonian fluid behaviour using the particle Reynolds number $R e_{p}=\frac{\rho_{f} v_{t} d_{p}}{\eta_{W}}$ in which $d_{p}$ is the particle diameter. The dashed line in Figure 4 is a fit to experimentally observed behaviour (Turton and Levenspiel, 1986) and $24 / R e_{p}$ is applicable for creeping flow $\left(R e_{p}<0.2\right)$. It can be seen that, for over 4 decades of $R e_{p}$ tested, the agreement with the Newtonian values is close when $R e_{p}$ is calculated using a local viscosity, itself calculated from a vectoral addition of the imposed shear rate and the falling particle shear rate, calculated as $v_{t} / d$. This is also the geometric mean of the belt shear rate and the shear rate due to sphere motion. The deviation from $24 / R e_{p}$ appears to be somewhat delayed for shear thinning materials compared to Newtonian fluids.

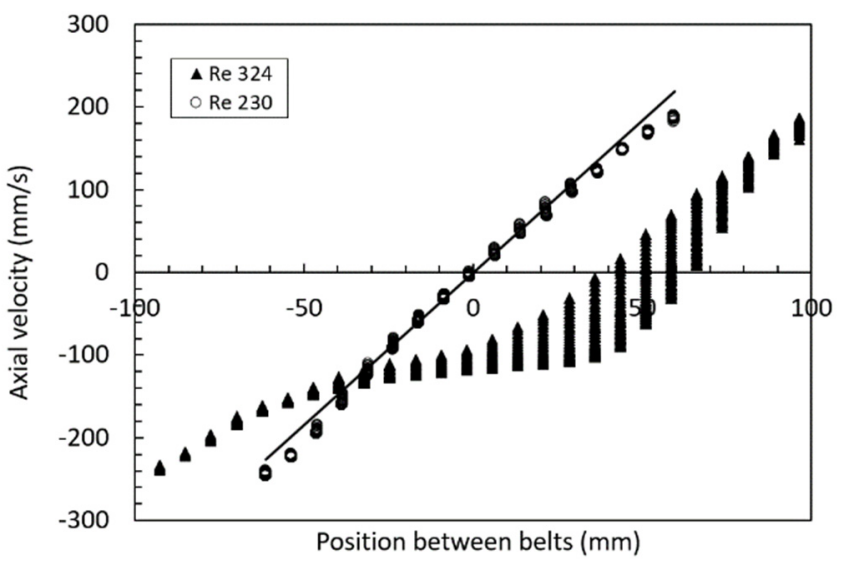

Fig. 3. PIV measurements horizontal mid-plane $(0.6 \mathrm{wt} \% \mathrm{CMC})$. Note the relatively stable, linear response at $R e<300$.

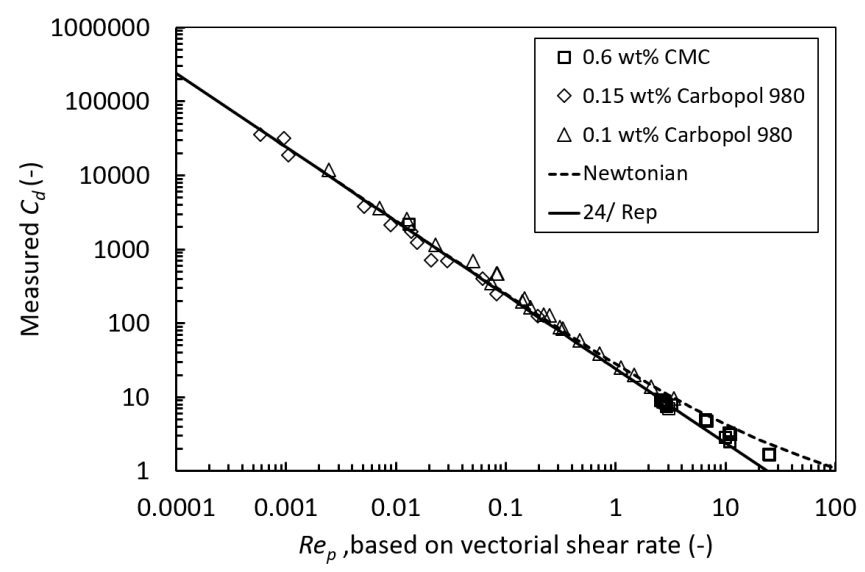

Fig. 4. Comparison of the particle drag coefficient, $C_{d}$, for nonNewtonian fluids to the expected behaviour for Newtonian fluids (line).

\section{Particle settling and particle acceleration}

When a particle is contained within a turbulent eddy it is subjected to rapid acceleration due to the difference between the mean local velocity and that characterising the eddy. The steady state terminal velocity, $v_{t}$, examined in the section above is a function of the various body forces, e.g. the buoyancy force, and the drag forces alone, but during acceleration there are several more forces to contend with, the complexity of which increases with $R e_{p}$. For in-elastic low Reynolds numbers flows, $\operatorname{Re}_{p}<1$, the full force balance on the particle is given by (e.g., Maxey and Riley, 1983) the Basset-Boussinesq-Oseen equation (BBO). The BBO equation expresses the acceleration of a particle in terms of the drag force, the force due to the pressure gradient (ignored here), the apparent mass due to the acceleration of the fluid by the particle, the Basset history force which accounts for changes in boundary layers and the applied body forces, which in this case reduce to the simple buoyancy force. Double integration of the BBO equation will produce the equation of the particle's trajectory with time. Although polymer solutions are generally elastic, the low polymer concentrations used in the work here meant that elastic effects were insignificant so that the BBO equation was still applicable. While it is likely that particles are subjected to higher accelerations for short periods in turbulent flow, the very low elasticity of these suspensions suggests that elastic effects will also be minimal for this more complex flow. 
To examine the effect of acceleration on settling behaviour the particles listed in Table 2 were accelerated by gravity through Newtonian (47 wt \% sucrose) and Carbopol solutions. Particles were allowed to settle in a tank of quiescent fluid and tracked using similar optical techniques to those employed for sheared flows (above). For the same particles travelling through Carbopol solutions the observed and calculated values are in substantial agreement when an arbitrary constant viscosity is used. Furthermore, for these particles, the difference between the trajectories calculated using just the drag forces and buoyancy forces and that calculated using the full $\mathrm{BBO}$ equation is very slight. A time varying viscosity can be calculated by estimating the shear rate due to the particle's motion. From the observations of particle motion in sheared suspensions, the shear rate due to the particle's motion may be approximated by particle velocity divide by particle diameter $\left(v_{t} / d_{p}\right)$. This value is then used to calculate the viscosity of the fluid surrounding the particle. Comparison of the calculated trajectories using this method and the experimental data is shown in Figure 5.

Figure 5 shows that using a viscosity calculated in this manner slightly underpredicts the acceleration of the particle but has the same form. This suggests that the calculated shear rate is too high. For the sheared settling experiments, the shear rate created by the particle motion is only a small fraction of the applied shear rate, and the flow field around the particle allows for a more uniform viscosity, approaching the Newtonian case. It can be inferred from the above that the BBO equation can be used in turbulent suspension calculations for non-Newtonian suspensions, and only drag and buoyancy forces need be considered. Further investigation of the behaviour of settling particles in sheared fluids using CFD can be seen in greater detail in Sethuraman et al. (2017) and Sethuraman (2020).

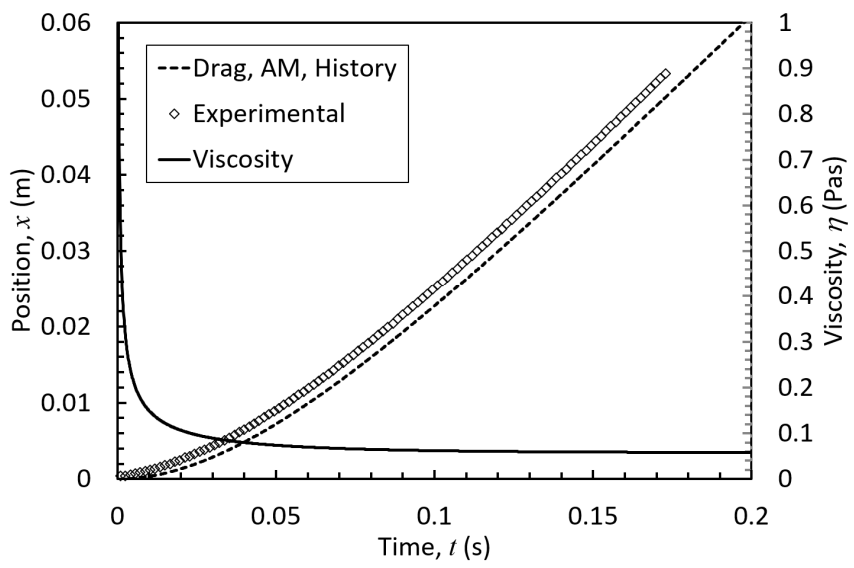

Fig. 5. Comparison of the observed particles trajectory settling in a non-Newtonian solution (symbols) with the calculated trajectory using the full $\mathrm{BBO}$ equation (line) based on a calculated local viscosity. The local viscosity is shown on the right axis.

\section{Instantaneous viscosity distribution}

Having established that particles settling in sheared nonNewtonian fluids behave in a very similar manner to those settling in Newtonian fluids providing the viscosity of the fluid local to the particle is used, it is then necessary to establish if and how the viscosity of the non-Newtonian fluid spatially varies in turbulent pipe flows.

The instantaneous viscosity of the fluid at any location within the pipe is obtained from the second invariant of the strain tensor, $S$, i.e. $\eta=\frac{\tau(\dot{\gamma})}{\dot{\gamma}} \quad \tau=\tau(\dot{\gamma}) \quad \dot{\gamma}=\sqrt{2 S_{i j} S_{i j}} \quad S_{i j}=\frac{1}{2}\left(\frac{d u_{i}}{d x_{j}}+\frac{d u_{j}}{d x_{i}}\right)$

where $\eta$ is the local viscosity, $\dot{\gamma}$ is the local shear rate, $\tau$ is the local shear stress, $S$ the strain rate tensor, and $u$ and $x$ are the velocities and directions, which for 1-D pipeline flow are simply the $\mathrm{x}$ and $\mathrm{y}$ coordinates in a transverse plane across the pipe. It should be noted that although the flows studied are steady state in terms of the mean velocity, the turbulent nature of the flow is time varying, and it is snapshot values of this varying strain that is used to obtain the average instantaneous viscosity, $<\eta>$, at a point in the flow.

For a Herschel-Bulkley fluid, say, the viscosity at the wall of the pipe is readily obtained from the wall shear stress, $\tau_{w}=\Delta p D / 4 L$ as

$$
\eta_{w}=k^{1 / n} \frac{\tau_{w}}{\left(\tau_{w}-\tau_{y}\right)^{1 / n}}
$$

where $\tau_{\mathrm{y}}, k$ and $n$ are the Herschel-Bulkley rheological parameters.

Analysing the data base from several DNS experiments of visco-plastic flows at wall Reynolds numbers similar to those found in hydraulic conveying systems, Rudman et al. (2004) demonstrated that the average instantaneous viscosity increases from $\eta_{w}$ to a maximum on the pipe centre line, or dynamic centre (Figure 6). In Figure 6, the viscosity distributions are shown for two flows of different generalized pipe Reynolds number $R e_{g}=\frac{\rho_{f} V D}{\eta_{W}}(V$ is the average velocity of flow $)$.

The DNS database, so created, provides estimates of the variation of the average values of shear stress, strain rate and viscosity within the pipe, which combined with the understanding of sheared settling can then be used to modify the model of the vertical concentration. However, the particle diffusivity, required by the model, is yet to be determined and must be extracted from experimental data as described in the next section.

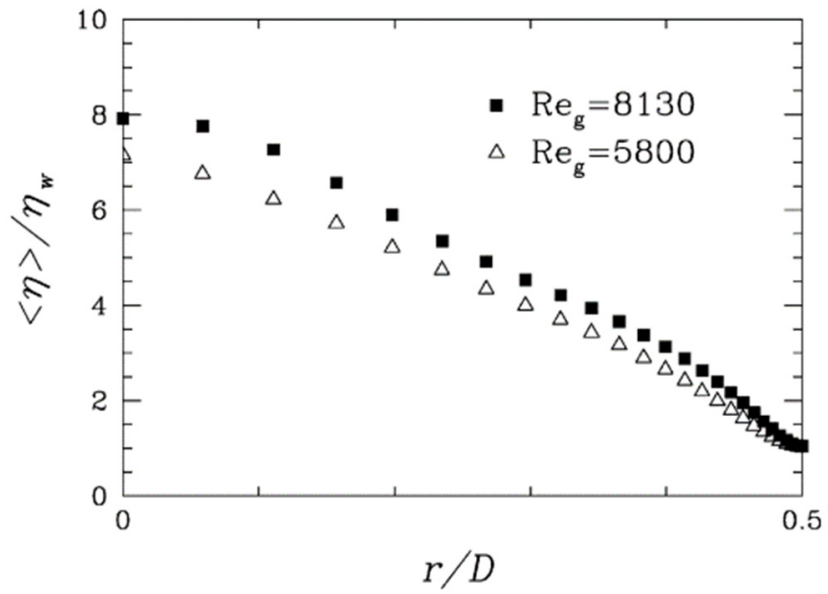

Fig. 6. Typical variation of normalized average instantaneous viscosity with pipe radius for flows of different generalized pipe Reynolds number (Rudman et al., 2004).

\section{Pipeline measurements}

Parallel experiments were conducted in the $50 \mathrm{~mm}$ pipe test facilities at the CSIRO in Melbourne and at the Czech Tech- 
nical University in Prague, using similar suspensions and techniques, but only the latter's facility and experiments conducted in that facility will be described here for brevity.

\section{Pipe test loop}

A fixed inventory pipe loop (Figure 7) is located in the Water Engineering Laboratory of the Czech Technical University in Prague. The total length of the rig is $23 \mathrm{~m}$ and the volume of its pipeline is 45 litres. The total length of the horizontal plexiglass section is $6.20 \mathrm{~m}$ and the internal diameter of its pipe is $50.0 \mathrm{~mm}$. In the horizontal pipe, a differential pressure is measured over Section 3, which is $1 \mathrm{~m}$ long. Differential pressures are also measured in two vertical sections (Section 1 and Section 2) of invert U-tube composed of pieces of the PPR pipe (pipe made of Polypropylene Random Copolymer) of the internal diameter $D=51.4 \mathrm{~mm}$. A Krohne Optiflux 5000 electromagnetic flow meter measures the flow rate in the upgoing leg of the vertical U-tube connected to the centrifugal pump discharge. The delivered concentration of the slurry, and hence the mass flux, is determined by analysis of the differential pressure transducer outputs from Sections 1 and 2.

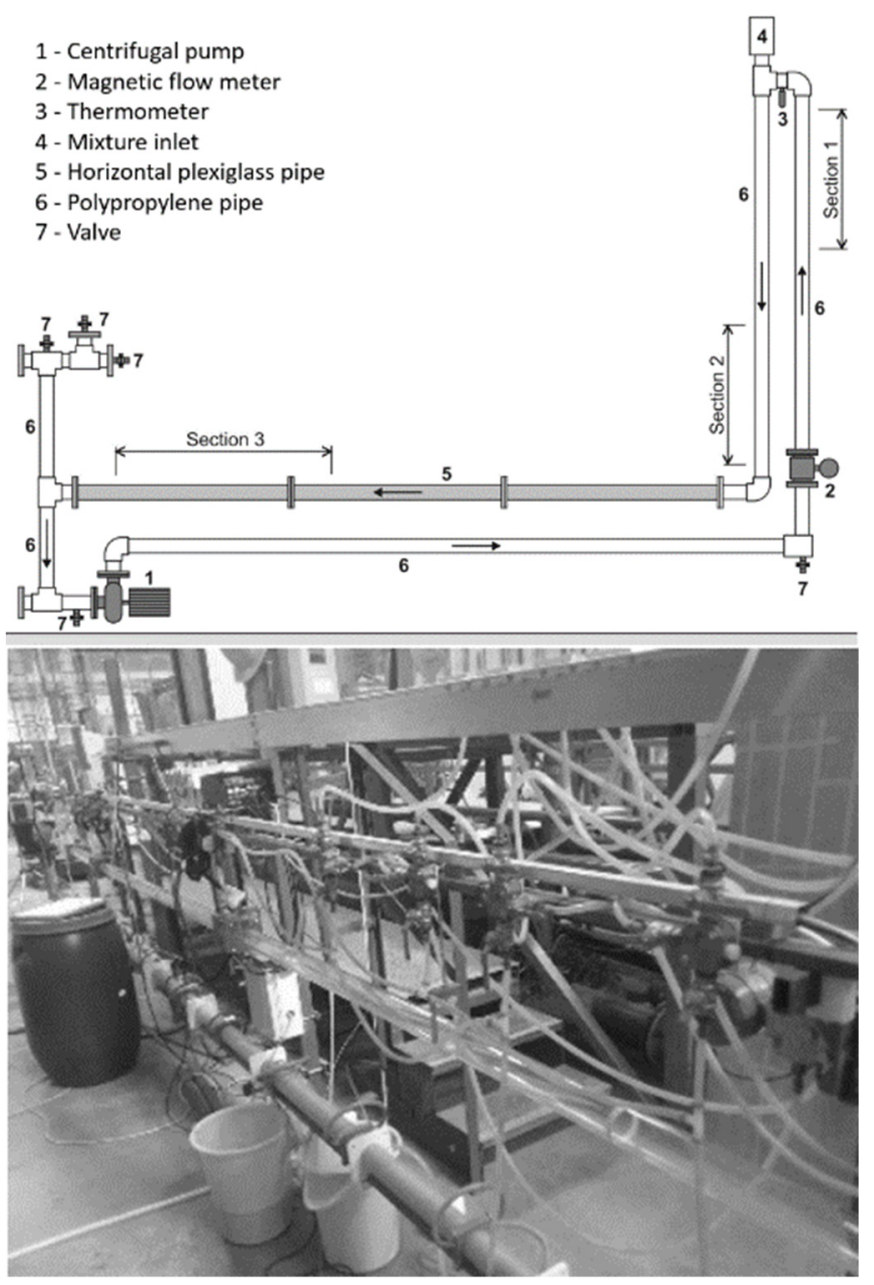

Fig. 7. Experimental setup at Czech Technical University in Prague.

Distribution of solids concentration are measured at various locations along the horizontal plexiglass section using an electrical resistivity tomographic (ERT) system constructed at
Cape Peninsula University of Technology (CPUT). The system comprises 3 separate rings, each ring having 16 electrodes, and senses a tomographic image of conductivity distribution simultaneously in up to three pipe cross sections. The rings are connected to a CPUT processing unit controlled by CPUT tomography software. Processed ERT data are further post-processed using EIDORS procedures (Lionheart et al., 1999).

The pump is driven by an electric motor with a variable frequency converter. The temperature of the flowing medium is measured in the vertical invert pipe.

\section{Materials}

For these tests only visco-plastic fluids were used, unlike in the settling tests where a wider range of rheological properties were examined. Two non-Newtonian carrier fluids made from aqueous solutions of Carbopol Ultrez 10 were used to transport mixtures of glass beads through the test rig. Two different solids were used, TK 1.5 and B7.

The TK1.5 material is effectively monodisperse with the median size $d_{50}=1.5 \mathrm{~mm}$. Sieving showed that all particle sizes fell between 1.49 and $1.61 \mathrm{~mm}$. The solid's density is $\rho_{\mathrm{s}}=2488$ $\mathrm{kg} / \mathrm{m}^{3}$. The concentration in a loose poured bed was $c_{\mathrm{b}}=0.61$, and the terminal settling velocity of individual beads in water $v_{t}=0.223 \mathrm{~m} / \mathrm{s}$.

The B7 material is also a narrow-graded material with particle sizes ranging from 0.4 to $0.7 \mathrm{~mm}$ with $d_{50}=0.57 \mathrm{~mm}$ and $d_{18}=0.47 \mathrm{~mm}, d_{84}=0.67 \mathrm{~mm}$. The density of particles is 2450 $\mathrm{kg} / \mathrm{m}^{3}$ and the terminal settling velocity of individual particles in water is $0.059 \mathrm{~m} / \mathrm{s}$.

Mixtures of glass beads and Carbopol solutions were tested in various combinations in the test loop. Figure 8 and Figure 9 show results of measurements of hydraulic gradient $i$ (the frictional pressure drop) over a range of flow velocities, $V$, for a mixture and their comparison with the calculated $i$ vs $V$ for the corresponding Carbopol carrier fluid. Each plot identifies the transition between the laminar and turbulent regime on the measured $i-V$ curve for the flow of the mixture. The two plots demonstrate results for two quite different types of mixtures. The mixture used for the data in Figure 8 contains a high volumetric concentration of glass beads $(30 \% \mathrm{v} / \mathrm{v}$ of $\mathrm{B} 7$ ballotini) in a relatively mildly viscous carrier fluid (low values of the yield stress and the other rheological parameters, see legend of Figure 8). For such a mixture, the presence of coarse particles increases flow resistance significantly in both laminar and turbulent regimes.

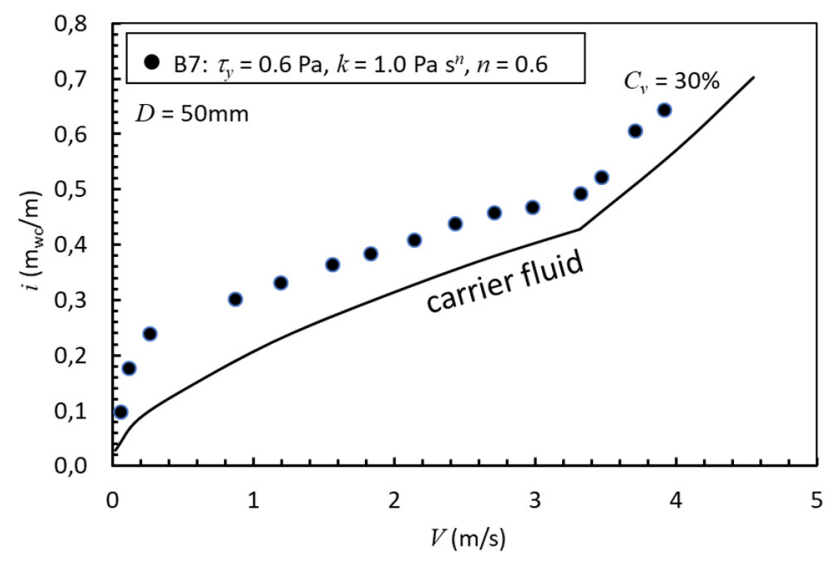

Fig. 8. Frictional pressure drop in $50 \mathrm{~mm}$-pipe for B 7 suspension. 


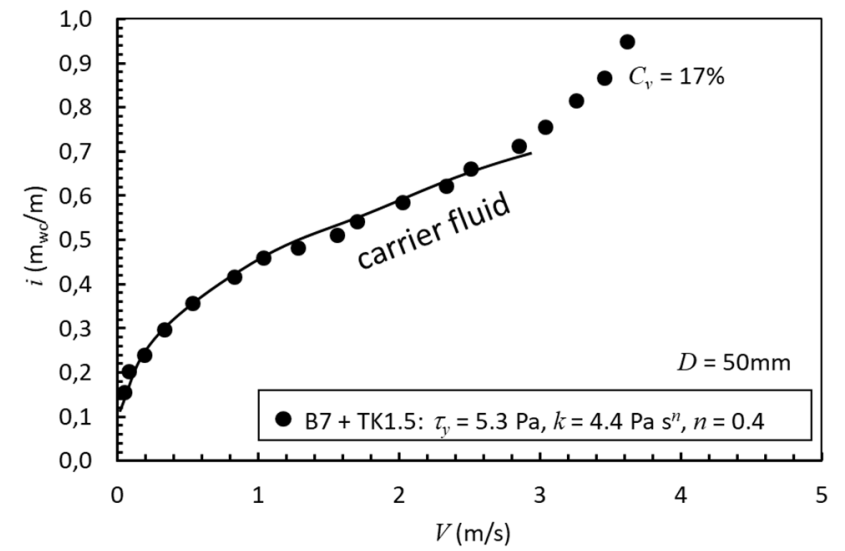

Fig. 9. Frictional pressure drop in $50 \mathrm{~mm}$-pipe for $\mathrm{B} 7+\mathrm{TK} 1.5$ suspension.

In Figure 9, the suspension has a significantly more viscous carrier and lower concentration of glass beads $(17 \% \mathrm{v} / \mathrm{v}$ mixture of TK1.5 and B7) which produces virtually negligible solids contribution to the total resistance in the laminar regime. The solids contribution is not negligible in the turbulent regime.

\section{Concentration distribution}

The two dimensional suspension conductivity maps, obtained using ERT can be converted into solids concentration maps, and these in turn can be analysed to give chord averaged concentration profiles, as shown in Figure 10 for the suspensions introduced in Figure 8 and Figure 9. These and similar profiles can then be used as test cases for the model predictions.

In general, the shapes of the measured concentration profiles exhibit small concentration gradients in the turbulent flow regime. The local gradients tend to be smaller in the core of the flow than outside the core. This indicates an existence of the effect of different local viscosity at different radial positions on the local concentration gradient. Due to a smaller instantaneous strain rate, the local viscosity is higher in the core of the flow than outside the core where the instantaneous strain rate is larger. The higher local viscosity decreases the settling capability of the conveyed grains and hence promotes local grain suspension in the core of turbulent flow.

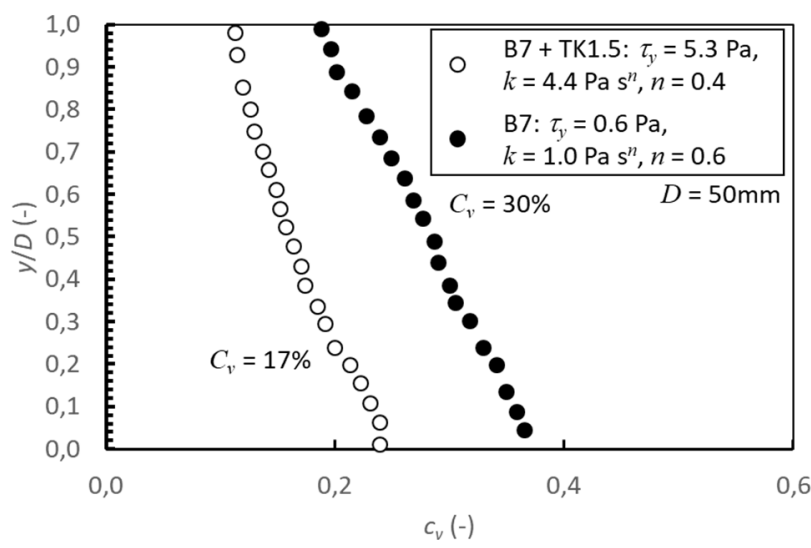

Fig. 10. Chord-averaged concentration profiles in turbulent flow obtained from measured ERT tomograms $\left(V=3.3 \mathrm{~m} / \mathrm{s}\right.$ and $R e_{g}=$ $2.2 \cdot 10^{3}$ for B7; $V=3.4 \mathrm{~m} / \mathrm{s}$ and $R e_{g}=2.3 \cdot 10^{3}$ for B7+TK1.5).

\section{Particle diffusivity}

Having now obtained some experimentally determined concentration profiles we are now in a position to extract some sample values of the particle diffusivity when the solids are conveyed by a non-Newtonian carrier.

Examination of the chord averaged laboratory concentration profiles and modifications applied to the Eskin model for particle diffusivity enabled Pěník et al. (2015) to extract the particle diffusivity profiles, using Eq. (1), for various pipeline velocities.

The following procedure was used to obtain the particle diffusivities from the measured concentration profiles:

1. The measured hydraulic gradient, $i$, is used to establish the wall shear stress, $\tau_{w}$.

2. The wall shear stress and rheological parameters are used to determine the apparent viscosity at the wall, Eq. (4).

3. The radial distribution of turbulent viscosity is obtained from the wall viscosity and imported viscosity-distribution function based on results of DNS-simulations of turbulent flows of Herschel-Bulkley fluids (Figure 6). The distribution is remapped to produce the chord-averaged values of the turbulent viscosity.

4. The local terminal settling velocity, $v_{t}$, is calculated as for Newtonian fluids but with the local turbulent viscosity replacing the Newtonian dynamic viscosity.

5. The measured local concentration gradient and the calculated local settling velocity are fed into the turbulentdiffusion formula Eq. (1) to obtain the local value of particle diffusivity.

6. The mean diffusivity is determined by integrating the flat distribution of diffusivity in the core of the turbulent flow and it is normalized by the wall shear velocity $U^{*}$ and the pipe radius $R$.

Average values of the particle diffusivity across the pipe normalised using the shear friction velocity and pipe radius, i.e. $\varepsilon_{s, \text { mean }} /\left(U^{*} R\right)$, are presented in Figure 11, where they are seen to be of the same order as those found in Newtonian fluids. This implies that using values similar to the Newtonian particle diffusivity in the model for non-Newtonian suspensions is a reasonable assumption. Furthermore, it also implies that any elastic effects for these suspensions are negligible, as surmized. The results support the idea that a more uniform distribution of particles in the non-Newtonian carrier than in the Newtonian carrier is due primarily to the much smaller settling velocity in the non-Newtonian case.

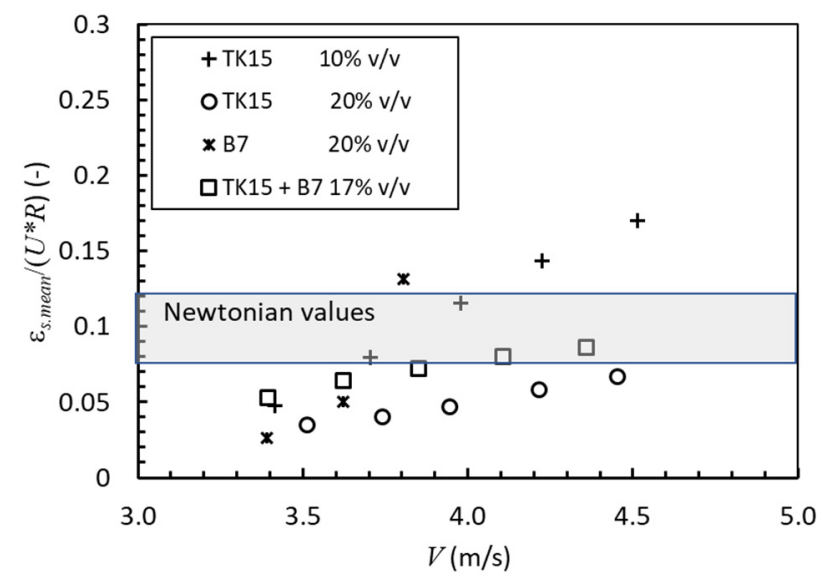

Fig. 11. Variation of normalised average particle diffusivity with pipe velocity for ballotini in Carbopol (Pěník et al., 2015.) 


\section{Comparisons}

To check the integrity of the modified Eskin model, predictions for some of the Newtonian case studies presented by Eskin are shown in Figure 12 along with those of the original model. Agreement is good, and if anything, the updated model predicts the lower concentration data somewhat better than the original model.

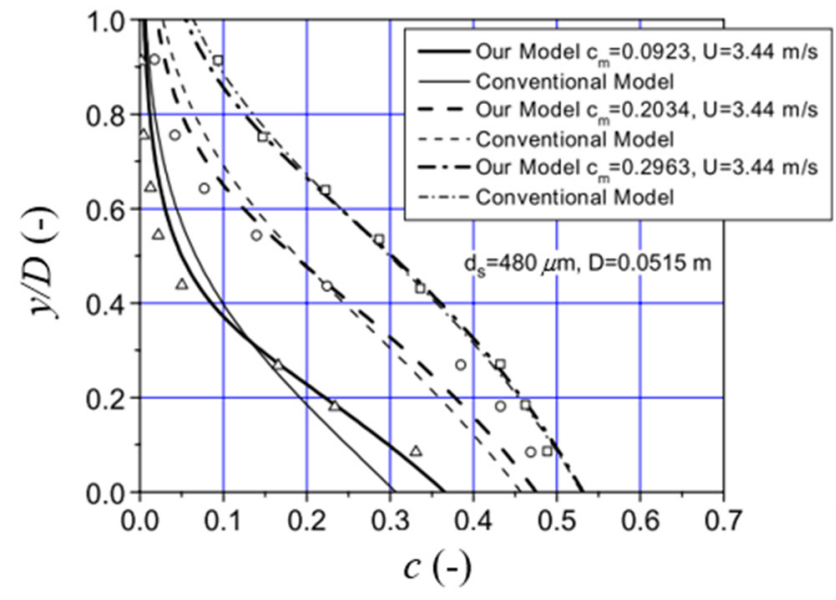

Fig. 12. Comparison between original Eskin model and the modified model presented here for Newtonian liquid-solid flow (particle diameter $d_{s}=0.48 \mathrm{~mm}$ ) at $V=3.44 \mathrm{~m} / \mathrm{s}$ and $R e_{g}=1.8 \cdot 10^{5}$ at various average particle volumetric concentrations $c_{m}$ for which the profiles were measured (circle, square, triangle) as reported by Eskin (2012).

The fully adapted model, using an instantaneous viscosity distribution similar to that shown in Figure 6, was used to predict the chord-averaged concentration profiles obtained in the laboratory. For comparison, predictions based on a Newtonian fluid, with the same wall viscosity, $\eta_{w}$, i.e. the same pipe Reynolds number were also made. Both sets of predictions are shown in Figure 13.

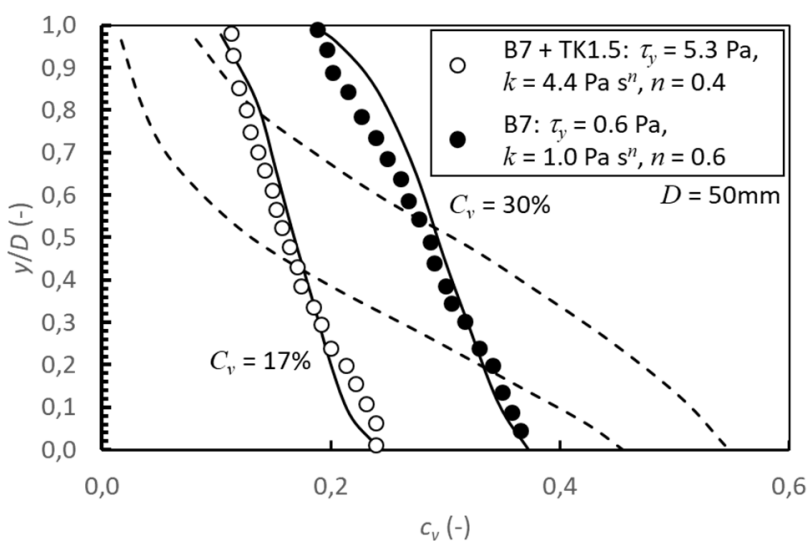

Fig. 13. Concentration profiles of ballotini suspended in a Carbopol solution (as in Figure 10) and their predictions. Predictions based on the non-Newtonian instantaneous viscosity distribution (solid line), and constant Newtonian viscosity distribution (dashed line) are shown for the same.

The agreement between the laboratory data and the modified model using the non-Newtonian behaviour and sheared settling is good, especially when compared with that of the equivalent Newtonian fluid. The effect of the increase in instantaneous viscosity with increasing distance from the wall, is seen to reduce particle migration, especially in the central core resulting in a more uniform solids concentration compared to that of the constant viscosity Newtonian case.

\section{CONCLUSIONS}

The concentration distributions of solids in moderate concentration, turbulent hydraulic Newtonian conveying systems are described well by models based on Rouse-Schmidt distributions. To assess whether such models could be successfully employed for turbulent non-Newtonian systems it was necessary to ascertain (i) how particles move in sheared nonNewtonian carrier fluids, (ii) how the fluid properties varied within the pipe and (iii) whether the particle diffusivity in such systems was comparable to those reported in Newtonian systems.

Experimental results, using a large scale planar Couette device demonstrated that, for the particle Reynolds numbers of interest, provided the viscosity of the non-Newtonian fluid, local to the particle was used, the particles not only had near identical settling behaviour to those in Newtonian fluids, but that they were also accelerated in a similar manner. The local viscosity of the fluid in which the particles were travelling was based on the local strain rate around the particles due to any applied shear, i.e. velocity profile, and that self-imposed by the fluids motion and the fluids constitutive relationships.

Based on results from computational studies, the variation of the instantaneous fluid viscosity within the pipe was estimated. This viscosity combined with the settling studies enabled the variation of particle settling velocity across the pipe to be established.

Experimental chord-averaged solids' concentration profiles, obtained for differing non-Newtonian turbulent suspensions, were then used, in conjunction with the settling velocity variation across the pipe, to extract the variation in particle diffusivity across the pipe, and establish average values. These average values were seen to be of the same order as those reported in the literature for Newtonian systems, which when combined with the other findings implied that providing local instantaneous viscosities were used, the chosen non-Newtonian systems behaved substantially the same as Newtonian systems.

A typical Rouse-Schmidt model implementation was modified to account for the change in viscosity, settling velocity and diffusivity, and predictions, so made, compared with experimental profiles of the turbulent non-Newtonian suspensions. There was a high degree of correlation between the model and the results, with the model capturing the prolonged solids residence in the central turbulent core, where the viscosity was the highest. This success was especially apparent when compared to a Newtonian model based on the same wall viscosity (i.e. same pipe Reynolds number) which predicted a far more pronounced concentration gradient in the pipe.

These results add support to the notion that previously established formulations used in hydraulic conveying design, based on water based systems, may be successfully employed providing the viscosities local to the particles, which take into account the shear field in the flow, are used.

\section{REFERENCES}

Cross, M.M., 1965. Rheology for non-Newtonian fluids: a new flow equation for pseudo-plastic systems. J. Colloid Sci., 20, 417-437.

Dou, H.S., Khoo, B.C., Yeo, K.S., 2007. Turbulent transition in 
plane Couette flows. In: Zhuang, F.G., Li. J.C. (Eds.): New Trends in Fluid Mechanics Research. Berlin, Heidelberg: Springer.

Eskin, D., 2005. An engineering model of solids diffusivity in hydraulic conveying. Powder Technology, 159, 2, 78-86.

Eskin, D., 2012. A simple model of particle diffusivity in horizontal hydrotransport pipelines. Chemical Engineering Science, 82, 84-94.

Gillies, R.G., 1993. Pipeline flow of coarse particles. Department of Chemical Engineering, University of Saskatchewan, Saskatoon.

Gillies, R.G., Shook, C.A., 1994.Concentration distributions of sand slurries in horizontal pipe flow. Particulate Science and Technology, 12, 1, 45-69.

Gnambode, P.S., Orlandi, P., Ould-Rouiss, M., Nicolas, X., 2015. Large-eddy simulation of turbulent pipe flow of power-law fluids. International Journal of Heat and Fluid Flow, 54, 196-210.

Kaushal, D.R., Seshadri, V., Singh, S.N., 2002. Prediction of concentration and particle size distribution in the flow of multi-sized particulate slurry through rectangular duct. Applied Mathematical Modelling, 26, 10, 941-952.

Lionheart, W.R.B., Arridge, S.R., Schweiger, M., Vauhkonen, M., Kaipio, J.P., 1999. Electrical impedance and diffuse optical tomography reconstruction software. In: The 1st World Congress on Industrial Process Tomography. Buxton, Derbyshire, UK, pp. 474-477.

Maxey, M.R., Riley, J.J., 1983. Equation of motion for a small rigid sphere in a nonuniform flow. Physics of Fluids, 26, 4, 883-889.

Missirlis, K.A., Assimacopoulos, D., Mitsoulis, E., Chhabra, R.P, 2001. Wall effects for motion of spheres in power-law fluids. Journal of Non-Newtonian Fluid Mechanics, 96, 459-471.

Pěník, V., Kesely, M., Matoušek, V., 2015. Coarse particle support in turbulent flow of visco-plastic carrier. In: Proc. Int. Conf. Experimental Fluid Mechanics 2015 - EFM15. Prague, pp. 588-592.

Rudman, M., Blackburn, H.M., Graham, L.J.W., Pullum, L., 2004. Turbulent pipe flow of shear-thinning fluids. Journal of Non-Newtonian Fluid Mechanics, 118, 1, 33-48.

Rudman, M., Blackburn, H.M., 2006. Direct numerical simulation of turbulent non-Newtonian flow using a spectral element method. Applied Mathematical Modelling, 30, 11, $1229-1248$.

Sethuraman, L., 2020. Particle settling in sheared nonNewtonian fluids. Monash University.

Sethuraman, L., Rudman, M., Gopalakrishnan, S., Bhardwaj, R., Chryss, A., Stephens, D., 2017. Predicting particle settling rate in a sheared mining slurry. In: The 18th International Conference on Transport and Sedimentation of Solid Particles. Prague.

Shook, C.A. Roco, M.C., 1991. Slurry Flow. Principles and Practice. Butterworth-Heinemann.

Singh, J., Rudman, M., Blackburn, H.M., Chryss, A., Pullum, L., Graham, L.J.W., 2016. The importance of rheology characterization in predicting turbulent pipe flow of generalized Newtonian fluids. Journal of Non-Newtonian Fluid Mechanics, 232, 11-21.
Turian, R.M., Yuan, T., 1977. Flow of slurries in pipelines. AlChE Journal, 23, 232-243.

Turton, R., Leverspiel, O., 1986. A short note on the drag correlation for spheres. Powder Technology, 47, 83-86.

Wilson, K.C., Thomas, A.D., 1985. A new analysis of the turbulent-flow of non-Newtonian fluids. Canadian Journal of Chemical Engineering, 63, 4, 539-546.

\section{NOMENCLATURE}

$A \quad$ Cross sectional area of the pipe $\quad\left(\mathrm{m}^{2}\right)$

c Local particle volumetric concentration (-)

$c_{b} \quad$ Loose poured volumetric concentration

$C_{d} \quad$ Drag coefficient of particle

$C_{v} \quad$ Average particle volumetric concentration

$C_{w} \quad$ Weight concentration

$d$ Gap width (m)

$d_{p} \quad$ Particle diameter $\quad$ (m)

$D \quad$ Pipe diameter (m)

$i \quad$ Hydraulic gradient $\quad(-)$

$K \quad$ Energy gradient $\quad(-)$

$k \quad$ Consistency coefficient $\quad\left(\mathrm{Pa} \mathrm{s}^{\mathrm{n}}\right)$

$L \quad$ Length of pipe section (m)

$m \quad$ Richardson and Zaki hindered settling exponent (-)

$n \quad$ Flow coefficient

$p$ Pressure $(\mathrm{Pa})$

$S \quad$ Strain rate tensor $\quad(1 / \mathrm{s})$

$R \quad$ Pipe radius (m)

Re Wall Reynolds number for planar Couette flow (-)

$R e_{g} \quad$ Generalized pipe Reynolds number

$R e_{p} \quad$ Particle Reynolds number

$r \quad$ Radial dimension (m)

$U^{*} \quad$ Shear velocity at wall $\quad(\mathrm{m} / \mathrm{s})$

$u \quad$ Local velocity $(\mathrm{m} / \mathrm{s})$

$V \quad$ Average velocity of flow $(\mathrm{m} / \mathrm{s})$

$v_{t} \quad$ Terminal settling velocity of particle $\quad(\mathrm{m} / \mathrm{s})$

$x \quad$ Dimension (m)

$y \quad$ Distance from bottom of pipe $\quad(m)$

$\dot{\gamma}$ Shear rate $(1 / \mathrm{s})$

$\mathcal{E}_{s} \quad$ Particle diffusivity $\left(\mathrm{m}^{2} / \mathrm{s}\right)$

$\eta \quad$ Viscosity (Pa s)

$\eta_{w} \quad$ Viscosity at the wall $\quad$ (Pa s)

$\rho$ Density $\left(\mathrm{kg} / \mathrm{m}^{3}\right)$

$\tau \quad$ Shear stress $\quad(\mathrm{Pa})$

$\tau_{w} \quad$ Shear stress at wall $\quad(\mathrm{Pa})$

$\tau_{y} \quad$ Yield stress parameter in flow model $\quad(\mathrm{Pa})$

CFD Computational Fluid Dynamics

CMC Carboxymethyl cellulose

CSIRO Commonwealth Scientific and Industrial Research Organisation

EIDORS Electrical Impedance Tomography and Diffuse Optical Tomography Reconstruction Software

Received 6 March 2021 Accepted 11 May 2021 